Article

\title{
Simplified Reactor Design for Mixed Culture-Based Electrofermentation toward Butyric Acid Production
}

\author{
Paola Paiano ${ }^{1,2}$, Giuliano Premier ${ }^{2}$ (), Alan Guwy ${ }^{2}$, Amandeep Kaur ${ }^{2}$, Iain Michie ${ }^{2}$, Mauro Majone ${ }^{1}$ \\ and Marianna Villano ${ }^{1, * \mathbb{B}}$ \\ 1 Department of Chemistry, Sapienza University of Rome, P.le Aldo Moro 5, 00185 Rome, Italy; \\ paola.paiano@uniroma1.it (P.P.); mauro.majone@uniroma1.it (M.M.) \\ 2 Sustainable Environment Research Centre (SERC), Faculty of Computing, Engineering and Science, \\ University of South Wales, Pontypridd, Mid-Glamorgan CF37 1DL, UK; \\ iano.premier@southwales.ac.uk (G.P.); alan.guwy@southwales.ac.uk (A.G.); \\ Amandeep.kaur@southwales.ac.uk (A.K.); iain.michie@southwales.ac.uk (I.M.) \\ * Correspondence: marianna.villano@uniroma1.it; Tel.: +39-06-49913651
}

Citation: Paiano, P.; Premier, G.; Guwy, A.; Kaur, A.; Michie, I.; Majone, M.; Villano, M. Simplified Reactor Design for Mixed Culture-Based Electrofermentation toward Butyric Acid Production. Processes 2021, 9, 417. https:// doi.org/10.3390/pr9030417

Academic Editor: Domenico Frattini

Received: 4 January 2021

Accepted: 14 February 2021

Published: 25 February 2021

Publisher's Note: MDPI stays neutral with regard to jurisdictional claims in published maps and institutional affiliations.

Copyright: (c) 2021 by the authors. Licensee MDPI, Basel, Switzerland. This article is an open access article distributed under the terms and conditions of the Creative Commons Attribution (CC BY) license (https:/ / creativecommons.org/licenses/by/ $4.0 /)$.

\begin{abstract}
Mixed microbial culture (MMC) electrofermentation (EF) represents a promising tool to drive metabolic pathways toward the production of a specific compound. Here, the MMC-EF process has been exploited to obtain butyric acid in simplified membrane-less reactors operated by applying a difference of potential between two low-cost graphite electrodes. Ten values of voltage difference, from $-0.60 \mathrm{~V}$ to $-1.5 \mathrm{~V}$, have been tested and compared with the experiment under open circuit potential (OCP). In all the tested conditions, an enhancement in the production rate of butyric acid (from a synthetic mixture of glucose, acetate, and ethanol) was observed, ranging from 1.3- to 2.7-fold relative to the OCP. Smaller enhancements in the production rate resulted in higher values of the calculated specific energy consumption. However, at all applied voltages, a low flow of current was detected in the one-chamber reactors, accounting for an average value of approximately $-100 \mu \mathrm{A}$. These results hold a substantial potential with respect to the scalability of the electrofermentation technology, since they pinpoint the possibility to control MMC-based bioprocesses by simply inserting polarized electrodes into traditional fermenters.
\end{abstract}

Keywords: single-chamber reactor; electrofermentation; applied voltage; mixed microbial cultures; butyric acid

\section{Introduction}

The ever-increasing attention toward the implementation of a circular bioeconomy has prompted investigations on the development of anaerobic mixed microbial culture (MMC) bioprocesses, whereby waste organic streams of complex and highly variable composition (e.g., agro-industrial wastewater, organic fraction of municipal solid waste, sewage) are converted into volatile fatty acids (VFA) via acidogenic fermentation [1-4]. Indeed, VFA are important precursors of numerous valuable end-products, such as platform chemicals (e.g., succinic acid), biopolymers (e.g., polyhydroxyalkanoates), and additives employed in several industrial sectors $[5,6]$.

However, a broad spectrum of products is typically attained from anaerobic MMCbased processes, resulting in relevant issues related to the separation and recovery of desired compounds. This, along with difficulties in controlling the fermentation pattern to maximize the yield of production of a desired end-product, hampers the diffusion of such bioprocesses at the industrial level. Attempts made so far to steer the spectrum of fermentation products have been mainly based on the possibility to control process parameters, such as the $\mathrm{pH}$ or temperature of the reaction medium, the hydrogen partial pressure, the hydraulic and solids retention time, as well as the feeding pattern (e.g., 
continuous versus batchwise strategy), although such strategies often lead to contrasting results [7-9].

A novel application of microbial electrochemical technologies, commonly referred to as "electrofermentation" (EF), has been more recently proposed to address this critical issue [10-12]. In particular, EF involves the use of electrodes to either supply electrons to or remove electrons from the metabolic fermentation process or to simply control the redox potential of the extracellular environment of reaction, thereby altering the intracellular ratio of the reduced and oxidized forms of nicotinamide adenine dinucleotide (NADH/NAD) and, in turn, affecting the rate and yield of NADH-dependent reactions [13]. This approach is extremely interesting, since both of the above-mentioned functions can be, in principle, finely tuned by using simple electrochemical systems [14]. Although the efficacy and viability of EF has been proven for a certain number of metabolic reactions, both involving pure and mixed microbial cultures, the underlying mechanisms, particularly at molecular and biochemical level, remain unclear [15-17].

As far as MMC-based EF is concerned, previous studies have provided straightforward indications that the utilization of a negatively polarized electrode (i.e., $-700 \mathrm{mV}$ vs. standard hydrogen electrode, SHE) can increase the rate and yield of butyric acid production during the acidogenic fermentation of a mixture of glucose, ethanol, and acetate [18]. A similar effect was observed even at a less negative potential of $-300 \mathrm{mV}$ vs. SHE, provided that a soluble redox mediator was concomitantly supplied along with the organic substrates [19]. The interest in butyric acid production relies on both its possible use for fuel production and its widespread application in several industrial fields, including food and beverage sectors, as well as pharmaceutical and chemical preparations (e.g., plastic materials or textile fibers) [20]. Presently, the production of butyric acid exclusively occurs via chemical synthesis, even though the use of biological processes is extensively encouraged [21], with the EF technology representing a very promising tool for this purpose. This is also because, as pointed out by the majority of above-mentioned studies $[10,11]$, while being the main driver of EF, the electric current consumed in the process is typically extremely low with respect to the rate of conversion of substrates. In principle, this finding holds remarkable advantages in terms of technology scalability and/or possibility to adapt existing anaerobic bioreactors into electrofermenters [22].

Notwithstanding these important aspects, a major challenge in the further development of EF remains the lack of simple, yet robust, reactor designs. Indeed, so far, electrofermentation experiments have been performed in lab-scale systems employing electrodes with the potential carefully controlled by a potentiostat. At the industrial scale, however, this approach may turn impractical due to problems with maintaining a set working electrode potential for large surface areas [23].

Additionally, with specific reference to butyric acid production, previous studies employed two-compartment reactors, whereby the EF process was carried out in a compartment containing the working electrode, which served as a cathode, and the other compartment containing the counter electrode, serving as an anode and catalyzing electrolytic oxygen evolution $[18,19]$. The two compartments were kept separated by expensive ion exchange membranes designed to permit selective ion flux between them while restricting oxygen diffusion, which is known to impair the metabolism of anaerobic microorganisms. In this context, the aim of the present study was twofold: (i) verify whether the stimulatory effect of an electrode on the MMC-based acidogenic production of butyric acid could be obtained by also using a simplified membrane-less bioreactor with the working and counter electrode placed in the same reaction environment, and (ii) to identify the optimal applied voltage between the working and counter electrode maximizing the rate of butyric acid production while minimizing energy consumption. 


\section{Materials and Methods}

\subsection{Inoculum}

The mixed microbial culture used as inoculum consisted of an anaerobic sludge collected from a full-scale anaerobic digester located in Cardiff (Wales, UK). In order to eliminate residual organic materials, before being used, the anaerobic sludge was settled and the supernatant replaced several times with anaerobic medium. The latter contained (g/L): $\mathrm{NH}_{4} \mathrm{Cl}(0.5), \mathrm{MgCl}_{2} \cdot 6 \mathrm{H}_{2} \mathrm{O}(0.1), \mathrm{CaCl}_{2} \cdot 2 \mathrm{H}_{2} \mathrm{O}(0.05), \mathrm{K}_{2} \mathrm{HPO}_{4}(8.7), \mathrm{KH}_{2} \mathrm{PO}_{4}(61.22)$, $10 \mathrm{~mL} / \mathrm{L}$ of a trace metals solution [24], and $1 \mathrm{~mL} / \mathrm{L}$ of vitamins solution [25]. The composition of the mineral medium allowed buffering to $\mathrm{pH} 5.5$ to inhibit the growth of methanogenic microorganisms. The microbial culture was maintained in a borosilicate glass bottle periodically flushed with $\mathrm{N}_{2}$ to ensure anaerobic conditions.

\subsection{Experimental Setup}

Simple glass bottles, with a total volume of $100 \mathrm{~mL}$, were used as membrane-less bioelectrochemical reactors. Each bottle was sealed with a rubber stopper equipped with two graphite rods ( $\approx 5 \mathrm{~cm}$ length, $6 \mathrm{~mm}$ diameter) and a Tygon (material nonpermeable to oxygen) sampling tube closed by a plastic clamp. The two electrodes were externally connected by a stainless steel wire and separated by two scaffolds, made by inert plastic material, in order to ensure a fix distance of $1 \mathrm{~cm}$ between the two rods (Figure 1a). Throughout the duration of the experiment, the reactors were maintained under magnetic stirring inside a temperature-controlled chamber, operating at $30^{\circ} \mathrm{C}$.

In detail, $17 \mathrm{~mL}$ of the anaerobic sludge was added to each reactor, which was then filled with $53 \mathrm{~mL}$ of mineral medium for a total liquid volume of $70 \mathrm{~mL}$. Upon preparation, all bottles were flushed with $\mathrm{N}_{2}$ to establish anaerobic conditions. In order to monitor the current generated in each system, a circuit of four parallel resistors $(10 \Omega)$ for each reactor was built and connected to the negative pole of the reactors. Resistance (R) and voltage (V) values were determined and logged every $60 \mathrm{~s}$ and elaborated in LabVIEW ${ }^{\mathrm{TM}}$ to calculate the current (I) that was flowing in the circuit, using the equation I =V/R (Ohm's Law).

Throughout the study, an adjustable constant voltage generated using a LabVIEW ${ }^{\mathrm{TM}}$ platform-based program was split into an array of several low-current voltage signals using the resistors. Operational amplifiers (Op-amps) were connected as voltage followers to assure supply the requisite current to overcome the limits of the I/O card used with LabVIEW $^{\mathrm{TM}}$. The Op-amp output voltages were applied to the whole array of reactors through the electrodes in each, so yielding applied potentials of incrementing values. The imposed signal was $-1.5 \mathrm{~V}$, and this voltage was the range over which the array of reactors was separated in steps of $100 \mathrm{mV}$ or higher (Figure 1b). As a consequence, several values of the applied potential difference between the electrodes were tested, ranging from $-0.60 \mathrm{~V}$ to $-1.5 \mathrm{~V}$. In addition, an open circuit potential (OCP) control experiment was performed in the absence of externally applied potential difference.

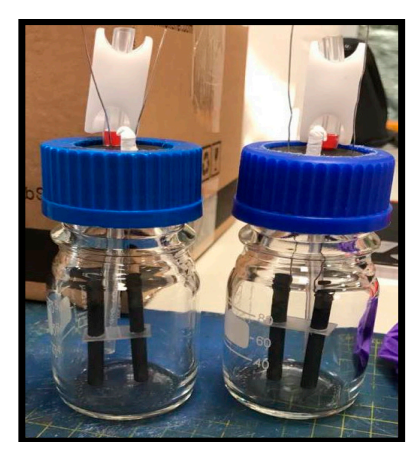

(a)

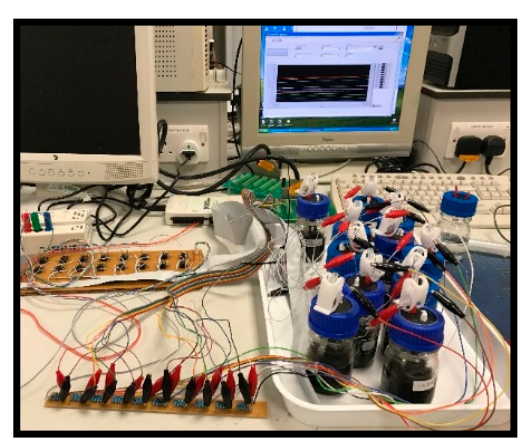

(b)

Figure 1. Picture (a) of single-chamber bioelectrochemical reactors used in the study. Experimental setup (b) adopted to apply the difference of potential between electrodes in each reactor. 
Experiments were performed by supplying glucose as either single substrate or in combination with ethanol and acetate. In both cases, the initial theoretical concentration of glucose was 1 gCOD (Chemical Oxygen Demand)/L, whereas an initial concentration of $0.30 \mathrm{gCOD} / \mathrm{L}$ was used for ethanol and acetic acid when the ternary mixture of substrates was used. Overall, the initial ratio between the concentration of microorganisms (as volatile suspended solids, VSS) and the concentration of substrates was set at approximately $0.15 \mathrm{gVSS} / \mathrm{gCOD}$. In particular, when glucose was used as single substrate, six values of applied potential (namely $-0.70 \mathrm{~V},-0.80 \mathrm{~V},-0.90 \mathrm{~V},-1.0 \mathrm{~V},-1.4 \mathrm{~V},-1.5 \mathrm{~V}$ ) in addition to the OCP condition were tested. Ten values of applied potential, separated in equal steps of $100 \mathrm{mV}$, were instead considered by using the mixture of substrates. In this case, the OCP condition and two out of the ten EF conditions (i.e., $-0.60 \mathrm{~V}$ and $-0.80 \mathrm{~V}$ ) were tested in triplicate.

\subsection{Analytical Methods and Calculations}

The concentration of microorganisms in the anaerobic sludge used as inoculum for the electrofermentation batch experiments was determined in terms of VSS, according to the standard method APHA [26].

The liquid phase of each reactor was periodically sampled for glucose, organic acids, and ethanol measurements. As for glucose, it was analyzed on filtered samples $(0.22 \mu \mathrm{m}$ porosity), according to the Sulfuric Acid-UV method [27], and its concentration was converted into COD based on the oxidation stoichiometry of $1.067 \mathrm{gCOD} / \mathrm{gGlucose}$. As for the determination of organic acids (i.e., acetic, propionic, isobutyric, and butyric acids) and ethanol, filtered samples $(0.22 \mu \mathrm{m}$ porosity) were injected $(1 \mu \mathrm{L})$ into a Dani Master (Milano, Italy) gas chromatograph (equipped with a $2 \mathrm{~m} \times 2 \mathrm{~mm}$ glass column packed with Carbopack) operated with He as carrier gas at $25 \mathrm{~mL} / \mathrm{min}$, the oven temperature at $175{ }^{\circ} \mathrm{C}$, and the flame ionization detector (FID) at temperature of $200{ }^{\circ} \mathrm{C}$. Measured concentrations were converted into COD according to the corresponding conversion factor (i.e., 1.067 gCOD/gAcetate, 1.51 gCOD/gPropionate, 1.82 gCOD/gButyrate, $1.82 \mathrm{gCOD} / \mathrm{gIsobutyrate}$, and $2.08 \mathrm{gCOD} / \mathrm{gEthanol})$.

A main parameter to evaluate the performance of the electrofermentation process was the rate of butyric acid production, which was calculated (as gCOD/Ld) through the linear regression of butyric acid concentration data (as the sum of the iso- and the normalisomers) versus time (d). Rates obtained in each condition were compared to the rate of production obtained in the OCP experiment.

In the tests performed in the presence of an externally applied voltage, data of the current were continuously recorded. These data were used to estimate the energy $(\mathrm{kJ})$ supplied to the system by integrating the current (A) over time (so to calculate the corresponding Coulombs $(C)$ ) and then multiplying $C$ for the applied voltage $(V)$. Therefore, the specific energy consumption associated to the production of butyric acid was determined by dividing the calculated values of supplied electrical energy by the amount of obtained butyric acid (gCOD) in each condition. From the calculated C, the cumulative electron equivalents (eq) transferred in each reactor were determined by using the Faraday's constant $(\mathrm{F}=96,485 \mathrm{C} / \mathrm{eq})$. Additionally, the equivalents released from glucose consumption were calculated by taking into account the molar conversion factor of 24 eq per mol of glucose.

\section{Results and Discussion}

\subsection{Electrofermentation of Glucose}

In the tests with glucose as the single carbon source (supplied at an initial concentration of $1 \mathrm{gCOD} / \mathrm{L}$ ), microbial fermentation resulted in a fast degradation of the substrate that was completely consumed in approximately one day (data not shown) and mainly converted into ethanol and acetic acid along with butyric acid, although the latter accumulated to a lower extent. For all the duration of the experiments (six days), the $\mathrm{pH}$ remained stable at an acidic value of about 5.7, as desired to inhibit the methanogenic activity. The obtained products accounted, in both OCP and EF experiments, for about $60 \%$ of the consumed COD, 
with the remainder being likely assimilated (i.e., used for microbial growth) or converted into unidentified products. In all EF experiments, a negative current flow was detected in the bioelectrochemical systems at values as low as few hundreds microamps, representing on average from $1 \%$ to $2 \%$ of the total consumed electron equivalents. Overall, no relevant effect due to the presence of polarized electrodes on the distribution of fermentation products was observed. This finding is, however, fully in agreement with what was previously noticed in dual-chamber experiments performed in the presence of glucose as the sole substrate, with or without the potential of the working electrode controlled at $-700 \mathrm{mV}$ (vs. SHE) [18].

3.2. Electrofermentative Conversion of a Synthetic Mixture of Glucose, Acetate, and Ethanol at an Applied Voltage of $-1.2 \mathrm{~V}$

The time course of the MMC anaerobic conversion of glucose when supplied together with ethanol and acetate is reported in Figure 2a, under both OCP and EF conditions, with main reference (for the sake of example) to the EF test performed by applying a difference of potential between the anode and the cathode electrodes of $-1.2 \mathrm{~V}$. In both operational conditions, glucose (ca. $0.90 \mathrm{gCOD} / \mathrm{L}$ ) was rapidly metabolized without any initial lagphase and completely depleted in approximately three days. As for acetate and ethanol, initially supplied at a concentration of about $0.30 \mathrm{gCOD} / \mathrm{L}$, their concentration increased during glucose consumption, whereas, once glucose was depleted, it slowly decreased over the remainder of the batch experiment, particularly in the OCP experiment. As for butyrate, its increasing trend is reported as the sum of the iso- and normal butyric acids, since in all conditions there was an initial production of iso-butyric acid which thereafter rapidly disappeared in favor of the production of the normal-isomer. Most likely, the latter reaction was catalyzed by microorganisms in the mixed culture, capable of bringing about the isomerization of butyric acid isomers [28]. However, under both EF and OCP conditions, butyrate started to accumulate from the beginning of the test and continued to be produced even after glucose was depleted. This latter finding, which is in line with the results of previous studies and with literature evidence, suggests that, upon glucose depletion, butyrate formation could be due to several factors, such as the condensation of ethanol and acetate $[19,29]$. Another explanation is related to the capability of anaerobic MMC to convert glucose into storage polymers, such as glycogen [30]. Moreover, during glucose consumption, lactate can also be produced, and it has been suggested that the transiently produced lactate, along with internal storage polymers, can be transformed into volatile fatty acids, such as butyric acid [31]. Throughout the duration of the two tests, the $\mathrm{pH}$ remained stable at an acidic value of 5.8. With reference to the EF condition, the trend of the electric current flowing in the circuit, reported in Figure 2b, shows that this parameter oscillated between $-35 \mu \mathrm{A}$ and $-130 \mu \mathrm{A}$, with an average value of $-80 \pm 15 \mu \mathrm{A}$. Such a relatively low electric current reflects the fact that the applied potential was well below the thermodynamic threshold of water electrolysis (i.e., 1.23 V) [32].

As a main result, by the end of the experiments, nearly twice as much butyrate was obtained under EF conditions relative to the OCP control (0.38 gCOD/L vs. 0.18 gCOD/L), thus clearly highlighting the stimulatory effect of EF on the enhanced production of this targeted compound.

Notably, a mass balance indicated that, while in the EF experiment at $-1.2 \mathrm{~V}$ applied voltage over $95 \%$ of the converted substrates could be recovered into fermentation products, a significant fraction (nearly 30\%) of the removed substrates could not be recovered in the OCP experiment (Figure 3 ). 


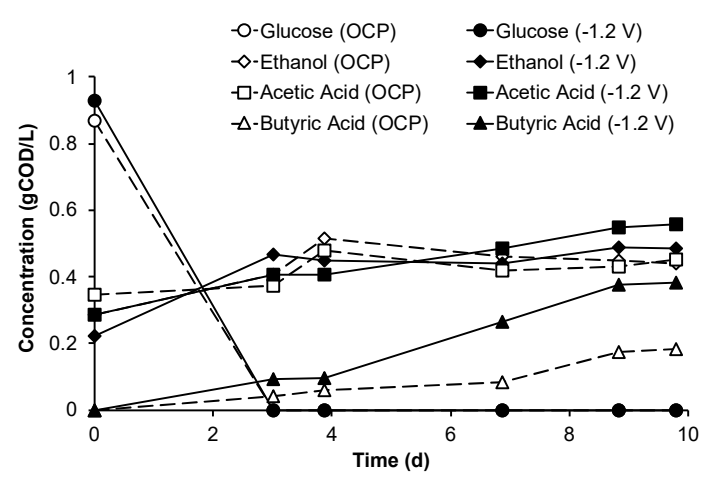

(a)

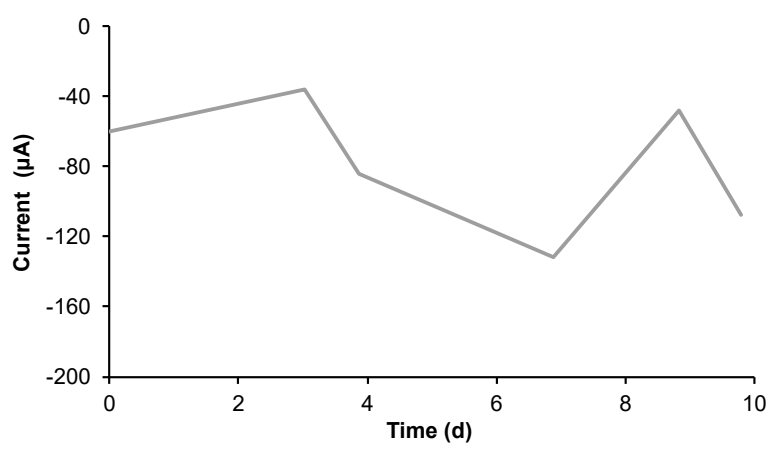

(b)

Figure 2. Time course of (a) mixed microbial culture (MMC) fermentation under open circuit potential (OCP) and electrofermentative $(\mathrm{EF},-1.2 \mathrm{~V})$ conditions and $(\mathbf{b})$ current in the EF test.

\section{口Unknown \$Acetic Acid $\square$ Ethanol $\square$ Butyric Acid}

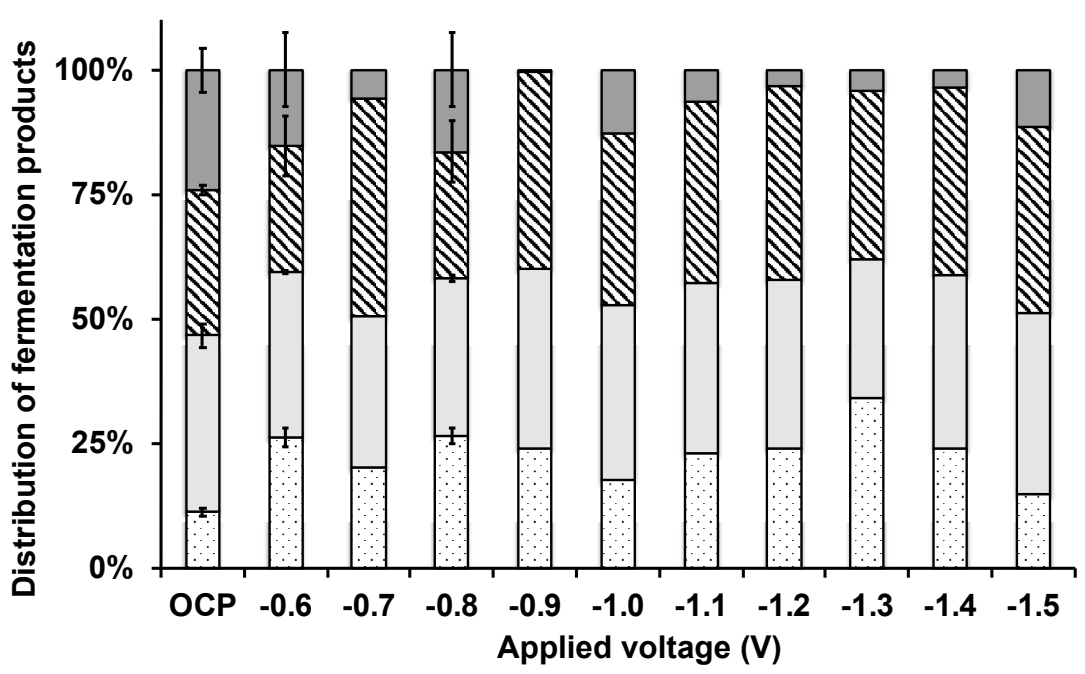

Figure 3. Percentage distribution of measured fermentation products calculated at the end of the batch experiments under OCP and EF conditions (error bars represent the standard error of replicated experiments).

\subsection{Effect of the Applied Potential on the Electrofermentation Process}

The MMC-based electrofermentation experiments with the synthetic mixture of substrates were carried out at different applied voltages, ranging from $-0.60 \mathrm{~V}$ to $-1.5 \mathrm{~V}$. Under all the tested EF conditions, the observed fermentation pattern was the same as the one described in paragraph 3.2 for the condition at an applied voltage of $-1.2 \mathrm{~V}$, and the removed substrates could be almost entirely (89-99\%) recovered into the measured fermentation products (Figure 3). Otherwise, in the absence of polarized electrodes (OCP control), the recovery was significantly lower, accounting for about $72 \%$.

Since a negligible amount of methane was produced in all conditions, it is possible that, limitedly to the OCP experiment, such a missing fraction of the converted COD was due to the formation of unidentified fermentation products, such as lactate (not identified by the used analytical techniques), hence raising the intriguing hypothesis that EF could also result in an abrupt shift in the fermentation pathway of the MMC [33]. This possibility certainly warrants further investigations to be confirmed.

A statistical analysis has been performed on replicated experiments in order to quantitatively assess the significance of differences observed among the tested conditions. In particular, an unpaired t-test analysis revealed that the difference in butyrate production between EF tests (at an applied voltage of $-0.60 \mathrm{~V}$ and $-0.80 \mathrm{~V}$ ) and the OCP control 
was statistically very significant $(p<0.005)$. Moreover, a very interesting finding is that, irrespective of the applied voltage, the rate of butyrate production was always substantially higher (up to 2.7 times) than that observed in the OCP control experiment (Figure 4a). The fold of enhancement of butyrate production rate, relative to the OCP control, apparently reduced to 1.3 when the applied potential was larger than $-1.4 \mathrm{~V}$.

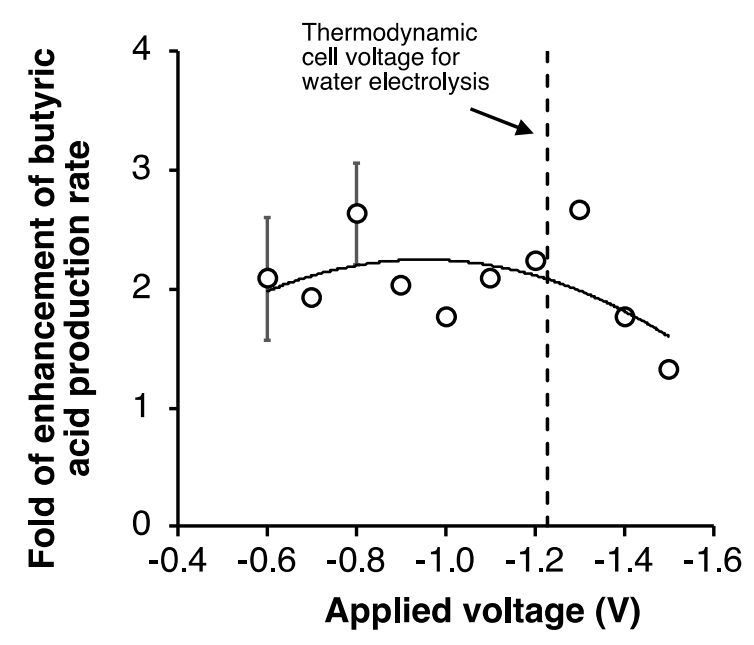

(a)

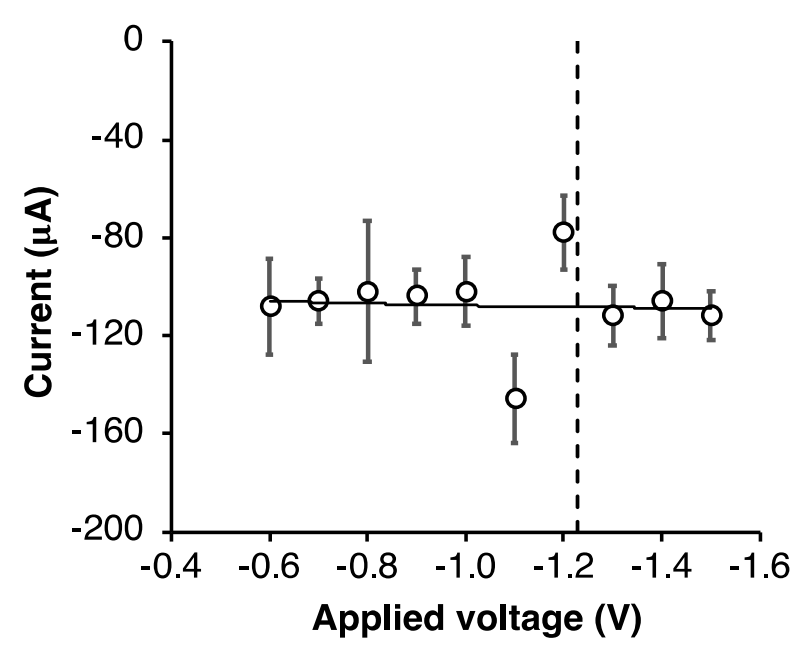

(b)

Figure 4. Effect of the applied voltage on (a) the fold of enhancement of butyric acid production rate relative to the OCP control (error bars represent the standard error of replicated experiments) and on (b) average electric current consumption (standard errors refer to mean values). Experimental data fitted with a 2nd order polynomial curve.

Figure $4 \mathrm{~b}$ reports the trend of the average values of electric current flowing in the circuit under the different conditions, which were all around $-100 \mu \mathrm{A}$. These very low values of current suggest that the onset of water electrolysis is unlikely, and therefore, the reduced butyrate production at $-1.5 \mathrm{~V}$ was not due to the adverse effect caused by oxygen evolution. Indeed, several studies have clearly indicated the occurrence of large overpotentials for hydrogen and oxygen evolution over graphite electrodes [34,35]. Most likely, this effect was due to a change in the redox potential of the reaction medium, which 
in turn may have affected the intracellular redox state of microbial cells impacting on butyrate production.

Electric current data were used to calculate the average energy consumption in each test and the corresponding specific energy consumption associated with butyric acid production at each applied voltage, as reported in Figure 5. As expected, the specific energy consumption increased as the cell voltage was increased (in absolute values). The highest and thus less favorable values $(>5.9 \mathrm{~kJ} / \mathrm{gCOD}$ ) were obtained when the applied voltage was larger than $-1.4 \mathrm{~V}$, also due to the lower production of butyric acids under such conditions. By contrast, substantially lower values (between 2.3 and $4.1 \mathrm{~kJ} / \mathrm{gCOD}$ ) were obtained in the other conditions, except for the applied voltage of $-1.1 \mathrm{~V}$ whose specific energy consumption was negatively affected by the apparently higher (in absolute values) value of the electric current. In addition, the values of electric current were used to calculate the cumulative charge consumed in correspondence of the time of glucose depletion in order to estimate the fraction of current contributing to the total electron consumption (i.e., deriving from electricity and organic substrates), which, under all the tested conditions, resulted in values ranging from approximately $2.3 \%$ to $4.3 \%$. Although quite low, these values are higher than those obtained in previous studies (accounting for approximately $0.5 \%$ ) performed by potentiostatically controlling the cathode potential in two-chamber systems [18]. Based on these considerations, future work will have to identify the optimal electrochemical strategy capable to further enhance butyric acid production in single-chamber reactors, as well as to establish the nature of reactions occurring at the electrodes, which resulted in relatively low electric current in absence of water electrolysis, and their impact on the electrofermentation process.

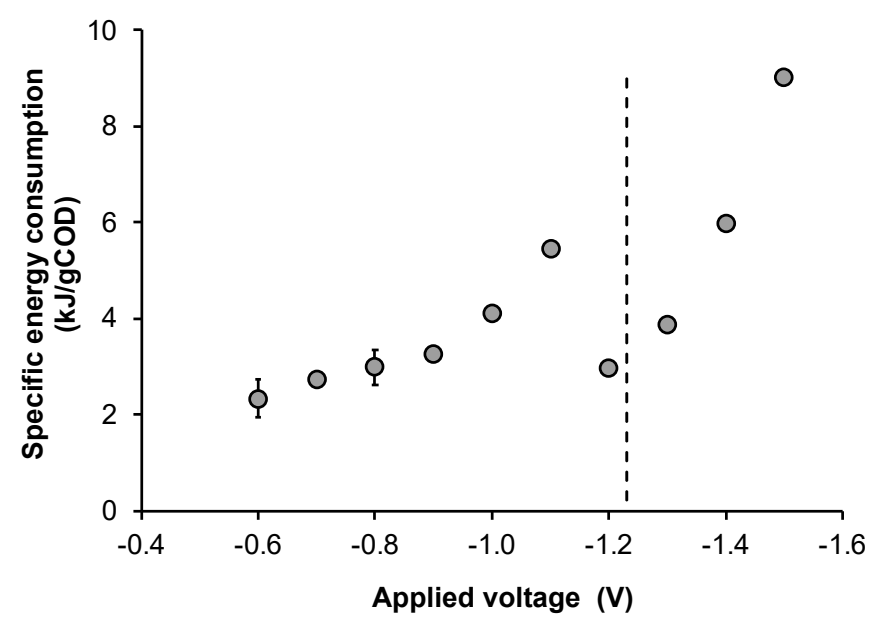

Figure 5. Effect of the applied voltage on the specific energy consumption associated to butyric acid production (error bars represent the standard error of replicated experiments). The dashed line represents the thermodynamic cell voltage for water electrolysis.

Taken as a whole, the obtained results allowed individuating a relatively wide range (from $-0.60 \mathrm{~V}$ to $-1.3 \mathrm{~V}$ ) of applied potential between the electrodes, wherein butyric acid production was significantly enhanced at the lowest observed values of specific energy consumption. These data are particularly interesting in view of a perspective scalability of the electrofermentation technology. Indeed, the possibility of simplifying the reactor design by removing the membrane as electrodes separator represents a key step to overcome scalability barriers [36,37]. This, along with the low current densities required to trigger the process, points to the possibility of directly inserting polarized electrodes of relatively small surface area into existing large-scale traditional MMC-based fermenters. Additionally, the process could be operated by either applying a voltage difference across a pair of electrodes (as in this study) or controlling the potential of an individual electrode by using a kit ad hoc designed to turn a bioreactor into a bioelectrochemical reactor, as previously described 
in the literature [22]. Possibly, all these improvements will contribute to rapidly drive the transition of the electrofermentation approach from the laboratory to the market.

\section{Conclusions}

The electrofermentation approach is concerned with the possibility of controlling microbial fermentation patterns by placing polarized electrodes within the reaction medium. This is interesting when dealing with undefined MMC, which typically generate a broad spectrum of fermentation products. Here, in order to further implement this promising technology, the MMC-based electrofermentation process toward butyric acid production has been studied in lab-scale single chamber, membrane-less reactors operated at ten different applied voltages (from $-0.60 \mathrm{~V}$ to $-1.5 \mathrm{~V}$ ). When a synthetic mixture of glucose, acetate, and ethanol was used as substrate, a significant enhancement in the production rate of butyric acid with respect to a control experiment (operated at open circuit potential, OCP) was observed in all the tested conditions. This enhancement (up to 2.7-fold increase) was most pronounced when the applied voltage was between $-0.60 \mathrm{~V}$ and $-1.3 \mathrm{~V}$, resulting in the lowest observed specific energy consumption for butyrate production. The less favorable condition was the one at an applied voltage of $-1.5 \mathrm{~V}$, whereby the rate of butyric acid production was still higher (1.3-fold) than in the OCP, but the corresponding specific energy consumption was significantly higher (ca. $9 \mathrm{~kJ} / \mathrm{gCOD}$ ) than in the other conditions. Notably, although this voltage $(-1.5 \mathrm{~V})$ is slightly higher that the theoretical voltage required for water electrolysis (i.e., $1.23 \mathrm{~V}$ ), the poorer performance was not due to oxygen evolution, as indicated by the very low current values (on average around $-100 \mu \mathrm{A}$ ) recorded in this and, more generally, in all the experiments.

These results assume a particularly interesting value if it is considered that they are completely in agreement with what was observed in dual-chamber reactors equipped with a membrane as ion separator, whereby an MMC fermentation pathway was driven in the presence of a potentiostatically controlled electrode potential. Further important experimental evidence is that, apparently, the electrofermentation process is independent of the origin and composition of the used MMC. This, along with the verified possibility to use membrane-less reactor configurations, holds promise in the perspective deployment of the technology, which could possibly exploit the opportunity to directly insert polarized electrodes into existing large-scale traditional MMC-based fermenters. Along this line, further research involving the use of real waste substrates of more complex composition is certainly warranted to confirm the practical potential of EF technology.

Author Contributions: Conceptualization, P.P., G.P., A.G., M.M., and M.V.; software, P.P., G.P., A.K., and I.M.; validation, P.P. and M.V.; formal analysis, P.P. and M.V.; investigation, P.P.; resources, G.P. and A.G.; data curation, P.P., A.K., I.M., and M.V.; writing-original draft preparation, P.P. and M.V.; visualization, P.P., G.P., A.G., I.M., M.M., and M.V.; supervision, G.P., M.M., and M.V.; project administration, G.P., A.G., M.M., and M.V.; funding acquisition, M.M. All authors have read and agreed to the published version of the manuscript.

Funding: This work was financially supported by the RES URBIS (RESources from URban BIo-waSte) Project (G.A. No 730349) in the frame of the European Horizon 2020 (Call CIRC-05-2016) program.

Institutional Review Board Statement: Not applicable.

Informed Consent Statement: Not applicable.

Data Availability Statement: Not applicable.

Acknowledgments: The authors wish to thank Hitesh Boghani for the skillful assistance with the experimental setup.

Conflicts of Interest: The authors declare no conflict of interest. 


\section{References}

1. Kleerebezem, R.; Joosse, B.; Rozendal, R.; Van Loosdrecht, M.C.M. Anaerobic digestion without biogas? Rev. Environ. Sci. Bio. Technol. 2015, 14, 787-801. [CrossRef]

2. Kircher, M. Bioeconomy-present status and future needs of industrial value chains. New Biotechnol. 2021, 60, 96-104. [CrossRef]

3. Salvador, R.; Puglieri, F.N.; Halog, A.; De Andrade, F.G.; Piekarski, C.M.; De Francisco, A.C. Key Aspects for Designing Business Models for a Circular Bioeconomy. J. Clean. Prod. 2021, 278, 124341. [CrossRef]

4. Agler, M.T.; Wrenn, B.A.; Zinder, S.H.; Angenent, L.T. Waste to bioproduct conversion with undefined mixed cultures: The carboxylate platform. Trends Biotechnol. 2011, 29, 70-78. [CrossRef]

5. Zhou, M.; Yan, B.; Wong, J.W.; Zhang, Y. Enhanced volatile fatty acids production from anaerobic fermentation of food waste: A mini-review focusing on acidogenic metabolic pathways. Bioresour. Technol. 2018, 248, 68-78. [CrossRef]

6. Valentino, F.; Morgan-Sagastume, F.; Campanari, S.; Villano, M.; Werker, A.; Majone, M. Carbon recovery from wastewater through bioconversion into biodegradable polymers. New Biotechnol. 2017, 37, 9-23. [CrossRef]

7. Bengtsson, S.; Hallquist, J.; Werker, A.; Welander, T. Acidogenic fermentation of industrial wastewaters: Effects of chemostat retention time and $\mathrm{pH}$ on volatile fatty acids production. Biochem. Eng. J. 2008, 40, 492-499. [CrossRef]

8. Jankowska, E.; Chwialkowska, J.; Stodolny, M.; Oleskowicz-Popiel, P. Volatile fatty acids production during mixed culture fermentation-The impact of substrate complexity and pH. Chem. Eng. J. 2017, 326, 901-910. [CrossRef]

9. Soomro, A.F.; Abbasi, I.A.; Ni, Z.; Ying, L.; Liu, J. Influence of temperature on enhancement of volatile fatty acids fermentation from organic fraction of municipal solid waste: Synergism between food and paper components. Bioresour. Technol. 2020, 304, 122980. [CrossRef]

10. Moscoviz, R.; Toledo-Alarcón, J.; Trably, E.; Bernet, N. Electro-Fermentation: How To Drive Fermentation Using Electrochemical Systems. Trends Biotechnol. 2016, 34, 856-865. [CrossRef]

11. Schievano, A.; Sciarria, T.P.; Vanbroekhoven, K.; De Wever, H.; Puig, S.; Andersen, S.J.; Rabaey, K.; Pant, D. Electro-FermentationMerging Electrochemistry with Fermentation in Industrial Applications. Trends Biotechnol. 2016, 34, 866-878. [CrossRef]

12. Chu, N.; Liang, Q.; Jiang, Y.; Zeng, R.J. Microbial electrochemical platform for the production of renewable fuels and chemicals. Biosens. Bioelectron. 2020, 150, 111922. [CrossRef]

13. Bhagchandanii, D.D.; Babu, R.P.; Sonawane, J.M.; Khanna, N.; Pandit, S.; Jadhav, D.A.; Khilari, S.; Prasad, R. A Comprehensive Understanding of Electro-Fermentation. Ferment. 2020, 6, 92. [CrossRef]

14. Sasaki, K.; Sasaki, D.; Kamiya, K.; Nakanishi, S.; Kondo, A.; Kato, S. Electrochemical biotechnologies minimizing the required electrode assemblies. Curr. Opin. Biotechnol. 2018, 50, 182-188. [CrossRef]

15. Toledo-Alarcón, J.; Fuentes, L.; Etchebehere, C.; Bernet, N.; Trably, E. Glucose electro-fermentation with mixed cultures: A key role of the Clostridiaceae family. Int. J. Hydrogen Energy 2021, 46, 1694-1704. [CrossRef]

16. Xafenias, N.; Anunobi, M.O.; Mapelli, V. Electrochemical startup increases 1,3-propanediol titers in mixed-culture glycerol fermentations. Process. Biochem. 2015, 50, 1499-1508. [CrossRef]

17. Kim, C.; Lee, J.H.; Baek, J.; Kong, D.S.; Na, J.-G.; Lee, J.; Sundstrom, E.; Park, S.; Kim, J.R. Small Current but Highly Productive Synthesis of 1,3-Propanediol from Glycerol by an Electrode-Driven Metabolic Shift in Klebsiella pneumoniae L17. ChemSusChem 2020, 13, 564-573. [CrossRef]

18. Villano, M.; Paiano, P.; Palma, E.; Miccheli, A.; Majone, M. Electrochemically Driven Fermentation of Organic Substrates with Undefined Mixed Microbial Cultures. ChemSusChem 2017, 10, 3091-3097. [CrossRef]

19. Paiano, P.; Menini, M.; Zeppilli, M.; Majone, M.; Villano, M. Electro-fermentation and redox mediators enhance glucose conversion into butyric acid with mixed microbial cultures. Bioelectrochemistry 2019, 130, 107333. [CrossRef]

20. Zigová, J.; Šturdík, E. Advances in biotechnological production of butyric acid. J. Ind. Microbiol. Biotechnol. 2000, 24, 153-160. [CrossRef]

21. Jiang, L.; Fu, H.; Yang, H.K.; Xu, W.; Wang, J.; Yang, S.-T. Butyric acid: Applications and recent advances in its bioproduction. Biotechnol. Adv. 2018, 36, 2101-2117. [CrossRef]

22. Rosa, L.F.M.; Hunger, S.; Gimkiewicz, C.; Zehnsdorf, A.; Harnisch, F. Paving the way for bioelectrotechnology: Integrating electrochemistry into bioreactors. Eng. Life Sci. 2017, 17, 77-85. [CrossRef]

23. Chiranjeevi, P.; Patil, S.A. Strategies for improving the electroactivity and specific metabolic functionality of microorganisms for various microbial electrochemical technologies. Biotechnol. Adv. 2020, 39, 107468. [CrossRef]

24. E Balch, W.; E Fox, G.; Magrum, L.J.; Woese, C.R.; Wolfe, R.S. Methanogens: Reevaluation of a unique biological group. Microbiol. Rev. 1979, 43, 260-296. [CrossRef]

25. Zeikus, J.G. The biology of methanogenic bacteria. Bacteriol. Rev. 1977, 41, 514-541.

26. APHA, AWWA, WEF Standard Methods for Examination of Water and Wastewater. Washingt. Am. Public Heal. Assoc. 2012.

27. Albalasmeh, A.A.; Berhe, A.A.; Ghezzehei, T.A. A new method for rapid determination of carbohydrate and total carbon concentrations using UV spectrophotometry. Carbohydr. Polym. 2013, 97, 253-261. [CrossRef]

28. Angelidaki, I.; Ahring, B.K. Isomerization ofn- andi-butyrate in anaerobic methanogenic systems. Antonie van Leeuwenhoek 1995, 68, 285-291. [CrossRef]

29. Reddy, M.V.; Mohan, S.V.; Chang, Y.-C. Medium-Chain Fatty Acids (MCFA) Production Through Anaerobic Fermentation Using Clostridium kluyveri: Effect of Ethanol and Acetate. Appl. Biochem. Biotechnol. 2018, 185, 594-605. [CrossRef] 
30. Carucci, A.; Lindrea, K.; Majone, M.; Ramadori, R. Different mechanisms for the anaerobic storage of organic substrates and their effect on enhanced biological phosphate removal (EBPR). Water Sci. Technol. 1999, 39, 21-28. [CrossRef]

31. Mulders, M.; Estevez-Alonso, A.; Stouten, G.R.; Tamis, J.; Pronk, M.; Kleerebezem, R. Volatile Fatty Acid Product Spectrum as a Function of the Solids Retention Time in an Anaerobic Granular Sludge Process. J. Environ. Eng. 2020, 146, 04020091. [CrossRef]

32. McHugh, P.J.; Stergiou, A.D.; Symes, M.D. Decoupled Electrochemical Water Splitting: From Fundamentals to Applications. Adv. Energy Mater. 2020, 10, 2002453. [CrossRef]

33. Moscoviz, R.; Trably, E.; Bernet, N. Electro-fermentation triggering population selection in mixed-culture glycerol fermentation. Microb. Biotechnol. 2017, 11, 74-83. [CrossRef]

34. Seehra, M.S.; Ranganathan, S.; Manivannan, A. Carbon-assisted water electrolysis: An energy-efficient process to produce pure $\mathrm{H}$ [sub 2] at room temperature. Appl. Phys. Lett. 2007, 90, 44104. [CrossRef]

35. Biswal, M.; Deshpande, A.; Kelkar, S.; Ogale, S. Water Electrolysis with a Conducting Carbon Cloth: Subthreshold Hydrogen Generation and Superthreshold Carbon Quantum Dot Formation. ChemSusChem 2014, 7, 883-889. [CrossRef]

36. Giddings, C.G.S.; Nevin, K.P.; Ewoodward, T. Simplifying microbial electrosynthesis reactor design. Front. Microbiol. 2015, 6, 468. [CrossRef]

37. Krieg, T.; Phan, L.M.P.; Wood, J.A.; Sydow, A.; Vassilev, I.; Krömer, J.O.; Mangold, K.-M.; Holtmann, D. Characterization of a membrane-separated and a membrane-less electrobioreactor for bioelectrochemical syntheses. Biotechnol. Bioeng. 2018, 115, 1705-1716. [CrossRef] 